\title{
Does symbolic and non-symbolic estimation ability predict mathematical achievement across primary school years?
}

\author{
Tatiana Tikhomirova ${ }^{1,2, *}$, Yulia Kuzmina ${ }^{1,2}$, and Sergey Malykh ${ }^{1,2}$ \\ ${ }^{1}$ Lomonosov Moscow State University, Department of Psychology, 119991 Moscow, Russia \\ ${ }^{2}$ Psychological Institute of Russian Academy of Education, 125009 Moscow, Russia
}

\begin{abstract}
The article presents the results of a longitudinal study of the association between number sense and success in learning mathematics in primary school. We analysed the data of 133 schoolchildren on two aspects of number sense related to the symbolic and non-symbolic magnitude estimation abilities and academic success in mathematics in third and fourth grade. The average age of schoolchildren during the first assessment was $9.82 \pm 0.30$; during the second assessment $-10.82 \pm 0.30$. For the analysis of interrelations, the cross-lagged method was used. It was shown that the reciprocal model best describes the data suggesting cross-lagged associations between number sense and the success in learning mathematics at primary school age. The results of the longitudinal analysis revealed differences in the relationship between the success in learning mathematics with the two aspects of number sense: academic success in third grade only predicted the indicator of number sense associated with the symbolic magnitude estimation ability in fourth grade. The differences in the age dynamics of the two aspects of number sense in primary school are also revealed: the indicator of number sense associated with the non-symbolic magnitude estimation ability was the most stable over time.
\end{abstract}

\section{Introduction}

The social and economical value of individual achievement in mathematics explains the research interest for the search of predictors of mathematical success among a range of cognitive characteristics $[1,2$, 3, etc.].

At the same time, number sense might be the most math-oriented and the least studied cognitive trait at least on the Russian sample $[4,5]$. Number sense is defined as an ability to estimate quantities wthout counting [4].

A number of studies showed that number sense is closely related to the success in learning mathematics $[6$, 7,8 , etc.]. In some studies these associations were found only at a certain age (for example, primary school age [9]), whereas others reported none $[10,11]$. These observed differences might be explained by the heterogenity of the psychological construct of number sense related to symbolically and non-symbolically expressed quantities $[4,12]$. These different aspects of number sense are in turn assessed with various test tasks. The aspects related to operating with symbolically expressed quantities are assessed with number stimuli [13], and the aspects related to operating with nonsymbolically expressed quantities are assessed with tests with stimuli consisting of various objects [8].

Despite the similarity of the two principles of quantity operating, the successful performance on the test tasks with symbolically and non-symbolically expressed quantities might vary for the same person which in turn might lead to differences in the association of number sense and mathematical achievememt [6]. This fact requires the assessment of both aspects of number sense - symbolic and non-symbolic - within one research programme.

To understand the mechanisms of the association of the analysed psychological constructs, it is crucial to assess the direction of this association which can only be done in a longitudinal study in which number sense and academic success would be measured in the same individuals across a certain time period [14, 15].

A number of longitudinal studies showed that individual differences in operating with non-sybolically expressed quantities at primary shool predicted success in learning mathematics at high school age $[3,16]$. At the same time, for number sense related to operating with symbolically expressed quantities, the results were different suggesting a bigger role of learning the concept of number and arithmetical operations. Individual differences in the ability to estimate the position of a number on the line in children of high school age have lower variance in comparison to children of primary school age [17], and children who got feedback during the test showed more precision in their performance than their peers who did not get any feedback [18].

A number of studies discussed age-related differences in the associations between symbolic and non-symbolic magnitude estimation observed during school mathematics education with development of the concept of the number and experience in operating with

*Corresponding author: tikho@mail.ru 
numbers [19]. Thus, a gradual improvement over the school-age period is observed for number sense associated with non-symbolic magnitude estimation [20]. At the same time, improvement in number sense measured with the Number Line test can occur spasmodically under a more significant influence of mathematics education [13].

The purpose of this study is to investigate the causal relationship between number sense and success in mathematics controlling for intelligence throughout the primary school age. In this case, we focused on two aspects of number sense reflecting the ability to operate with both symbolic and non-symbolic quantities. Participation of schoolchildren enrolled in primary school when the most intensive training on the concept of number and related operations occurs offers an opportunity to assess how the learning process mediates the association between number sense and success in mathematics.

\section{Methods}

\subsection{Participants}

The sample included schoolchildren from municipal general education institution participating in the Russian longitudinal study of the academic success of schoolchildren.

In the present work, the data of 133 students collected at the end of third and fourth grades were analysed. The average age of participants in third grade was 9.82 years (standard deviation $=0.30,45.1 \%$ boys), in the fourth grade it was 10.82 years (standard deviation $=0.30,45.1 \%$ boys $)$.

The analysis was carried out on the basis of anonimous personal data.

\subsection{Measures}

\subsubsection{Number sense, non-symbolic magnitude estimation ability}

In the Number Sense Task, yellow and blue dots different in size are presented on the screen [21]. The children were asked to decide if the array contains more yellow or blue dots, and press the corresponding key on the keyboard (Y or B). The stimuli contained 150 static images with arrays of yellow and blue dots, the number of which varies from 5 to 21 points of each color; the ratio of dots of two colors is either $1: 3$ or $6: 7$. The image appears on the screen for $400 \mathrm{~ms}$, maximum response time is 8 seconds. The test consists of three blocks of 50 tasks. The program records the accuracy of responses and response time.

\subsubsection{Number sense, symbolic magnitude estimation ability}

In the Number Line test, a line with the minimum and maximum values of 0 and 1000 , respectively, is displayed on the screen as well as a certain number at the top of the screen [21]. Students were asked to place the number displayed at the top of the screen on the line. Overall, there are 22 numbers in this task which should be placed on the line with the help of a computer mouse. The program registers the average deviation of the position marked by a student on the line from the actual position of each number. Thus, the higher is the value of the indicator for this test, the worse is the individual result.

\subsubsection{Intelligence}

In the Standard Progressive Matrices test, the tasks are grouped into 5 series, each of which consists of 12 tasks [22]. The number of correctly solved tasks for each series and the total score for the whole test are calculated.

\subsubsection{Math achievement}

Term grades for mathematics given by a primary school teacher were used as indicators of the success in learning. In the statistical analysis we used the arithmetic mean of the term grades.

\subsubsection{Statistical Approach}

First, a correlation analysis of the indicators of number sense, nonverbal intelligence and the success in learning mathematics assessed at the end of third and fourth grades was carried out. Spearman correlation coefficients were calculated.

Second, cross-lagged modeling was performed to analyze the longitudinal structure of the cause-effect relationships between the indicators of number sense and success in mathematics measured twice during the primary school age while controlling for intelligence.

Three types of associations were evaluated during the analysis: 1) autoregressive associations reflecting the stability of each trait over time, 2) contemporaneous associations that estimate the total variation of variables within each of the two assessments, and 3) cross-lagged associations showing how the variation of the preceding assessment of one trait explains the variation of the subsequent assessment of another trait.

We consistently compared four competing models of the relationship of the two aspects of number sense and the success in learning mathematics.

Model 1, autoregressive, assumed the connections between number sense and success in learning mathematics only within each time point (M1).

Model 2 assumed that the success in learning mathematics in the fourth grade depended on individual differences in number sense in third grade (M2).

Model 3, on the contrary, assumed that the successful performance on number sense test tasks depended on the successful learning of mathematics in third grade (M3).

Model 4, reciprocal, assumed that the success in mathematics measured in third grade predicted the success in number sense test in fourth grade, and conversely, number sense measured at an earlier age predicted success in mathematics at a later age (M 4). 


\section{RESULTS}

Table 1 presents the descriptive statistics for the indicators of number sense related to operating with arrays of objects (Number Sense) and estimating the position of a number on the line (Number Line), nonverbal intelligence (Standard Progressive Matrices) and success in learning mathematics.

Table 1. Descriptive statistics of the analysed indicators measured in third and fourth grade.

\begin{tabular}{|c|c|c|c|}
\hline Grade & Indicator & Mean & SD \\
\hline \multirow{4}{*}{$3^{\text {rd }}$} & Number Sense & 95.66 & 13.33 \\
\hline & Number Line & 93.44 & 68.93 \\
\hline & $\begin{array}{l}\text { Success in } \\
\text { learning } \\
\text { mathematics }\end{array}$ & 4.17 & 0.61 \\
\hline & $\begin{array}{l}\text { Standard } \\
\text { Progressive } \\
\text { Matrices } \\
\end{array}$ & 40.63 & 7.86 \\
\hline \multirow{3}{*}{$4^{\text {th }}$} & Number Sense & 100.13 & 14.25 \\
\hline & Number Line & 77.80 & 51.30 \\
\hline & $\begin{array}{l}\text { Success in } \\
\text { learning } \\
\text { mathematics }\end{array}$ & 4.17 & 0.63 \\
\hline
\end{tabular}

In Table 1, the average number of correct answers is indicated for the Number Sense Task and Standard Progressive Matrices tests, with a maximum of 150 and 60 tasks, respectively. The average deviation of the marked position of the number from the actual position was indicated for the Number line test. In this case, a lower value corresponds to a better, more accurate performance. The success in learning mathematics is estimated as the average value of term grades and varies from 2 to 5 .

According to Table 1, throughout primary school, number sense improved with a decrease in the standard deviation. On the contrary, the success in learning mathematics did not change while maintaining its variability.

\subsection{Correlation analysis: association between number sense and success in learning mathematics in third and fourth grades}

The correlation analysis focused on the structure of the relationship of the two aspects of number sense (Number Sense test - NS, and Number Line test - NL), nonverbal intelligence (Int, Standard Progressive Matrices) and success in learning mathematics (Math) in third and fourth grades. Table 2 presents Spearman correlation coefficients for the analysed indicators $\left({ }^{*} p<0.05\right.$,
$* * p<0.01)$ measured in third (lower left side) and fourth grades (upper right side).

Table 2. Results of correlation analysis of indicators measured in third and fourth grade.

\begin{tabular}{|c|c|c|c|c|}
\hline & NS & NL & Math & Int \\
\hline NS & 1 & $-0.41^{* *}$ & $0.33^{* *}$ & $0.30^{* *}$ \\
\hline NL & $-0.45^{* *}$ & 1 & $-0.42^{* *}$ & $-0.23^{*}$ \\
\hline Math & $0.38^{* *}$ & $-0.40^{* *}$ & 1 & $0.42^{* *}$ \\
\hline Int & $0.24^{*}$ & $-0.33^{* *}$ & $0.45^{* *}$ & 1 \\
\hline
\end{tabular}

According to Table 2, moderate significant correlations (from 0.33 to $0.45, p<0.01$ ) are observed between the indicators of cognitive development and the success in learning mathematics.

Comparative analysis of the correlation coefficients obtained in third and fourth grades showed that the association of number sense related to non-symbolic magnitude estimation with the success in learning mathematics in fourth grade becomes less pronounced in comparison with the third grade $(0.33$ vs. $0.38, p<0.01)$. The correlation between the two analyzed aspects of number sense is more pronounced in third grade $(\mathrm{r}=0.45, p<0.01)$ compared to fourth grade $(\mathrm{r}=0.41$, $p<0.01)$.

In general, according to the correlation analysis, the structure of the interrelationships between the indicators of cognitive development and the success in learning mathematics in third and fourth grade remains unchanged.

\subsection{Cross-lagged model: cause-effect relationship of number sense and success in learning mathematics}

In the cross-lagged analysis, four competing models of the relationship between the two aspects of number sense and the success in learning mathematics were compared controlling for nonverbal intelligence at primary school age.

Table 3 shows the fit of the four tested models with empirical data: M1 - autoregressive; M2 - number sense in third grade predicts the success in learning mathematics in fourth grade; M3 - success in learning mathematics in third grade predicts number sense in fourth grade; M4 - reciprocal.

According to Table 3, the reciprocal model (M4) best describes the empirical data suggesting that number sense measured in third grade predicts success in learning mathematics in fourth grade, and vice versa, success in mathematics in third grade predicts number sense in fourth grade.

The estimation of cross-lagged associations showed that the relationship between the success in learning 
mathematics in third grade and number sense in the Number Line task $(-0.33, p<0.05)$ was statistically significant. In other words, none of the aspects of number sense measured in the third grade significantly contributed to the variation in the success in learning mathematics by the end of primary school $(p>0.05)$.

Table 3. Fit indices for models of the relationship between number sense and success in learning mathematics.

\begin{tabular}{|c|c|c|c|c|c|}
\hline \multicolumn{2}{|c|}{ Indices } & M 1 & M 2 & M 3 & M 4 \\
\hline \multirow{3}{*}{$\underset{\frac{U}{u}}{U}$} & AIC & 3663 & 3666.2 & 3656.6 & 3659.9 \\
\hline & BIC & 3725.8 & 3739.4 & 3728.8 & 3738.4 \\
\hline & $\begin{array}{l}\text { adj. } \\
\text { BIC }\end{array}$ & 3650 & 3650.9 & 3641.4 & 3643.6 \\
\hline \multirow{3}{*}{ 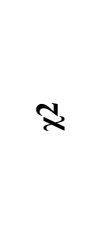 } & value & 16.9 & 12.09 & 2.5 & 1.79 \\
\hline & $\mathrm{df}$ & 9 & 5 & 5 & 3 \\
\hline & $\begin{array}{r}p- \\
\text { value }\end{array}$ & 0.049 & 0.033 & 0.78 & 0.62 \\
\hline \multirow{2}{*}{$\sum_{\simeq}^{\sqrt[\pi]{0}} \stackrel{0}{0}$} & Est. & 0.093 & 0.118 & 0.00 & 0.00 \\
\hline & $\begin{array}{r}90 \% \\
\text { CI }\end{array}$ & $\begin{array}{c}0.002- \\
0.16\end{array}$ & $\begin{array}{c}0.03- \\
0.21\end{array}$ & $\begin{array}{c}0.00- \\
0.09\end{array}$ & $\begin{array}{c}0.00- \\
0.04\end{array}$ \\
\hline \multirow{2}{*}{$\bar{E}$} & CFI & 0.98 & 0.98 & 1.00 & 1.00 \\
\hline & TLI & 0.95 & 0.92 & 1.03 & 1.01 \\
\hline
\end{tabular}

Note: AIC - Akaike Information Criterion; BIC - Bayesian information criterion; adj. BIC - sample-size adjusted BIC; $\chi^{2}$ (value, $d f, p$ ) - chi-square test (value, degrees of freedom, pvalue);RMSEA (Est., CIs) - root mean square error of approximation (value, $90 \%$ confidence intervals); CFI comparative fit index; TLI-Tucker-Lewis index

Among the contemporaneous associations, statistically significant connections between all the variables were obtained only at the first assessment while at the second assessment a statistically significant relationship was found only between the two aspects of number sense. At the same time, the correlation analysis performed on the data of fourth grade revealed moderate associations of number sense with the success in learning mathematics $(0.33<|\mathrm{r}|<0.42)$. In addition, a high regression coefficient between two assessments of the success in learning mathematics $(0.96, p<0.05)$ is noteworthy, which might cause distortion of contemporaneous associations [15].

Due to high temporal stability of the success in learning mathematics based on teacher evaluations, two modified theoretical models were examined to estimate cross-lagged associations. In these models, according to the principles of structural modeling, the success in learning is represented by one assessment - only in third or only in fourth grade. This method allowed us to evaluate the cross-lagged relationship between number sense and academic success taking into account the high temporal stability of the success in learning mathematics.

According to the analysis, both modified models have similar indices which confirms their good fit to the empirical data (RMSEA $\leq 0.06,0.00 \leq 90 \%$ CI $<0.08$; $\mathrm{CFI}>0.95$; $\mathrm{SMRM} \leq 0.06$; TLI $>0.90)$. The estimation of cross-lagged associations showed that the greatest regression weight is the connection, according to which the success in learning mathematics in the third class contributes to individual differences in the feeling of the number measured by the "Number line" test in the fourth class $(\beta=-0.33)$.

Thus, even with the high temporal stability of academic success, it was shown that learning mathematics in third grade is the most important factor in shaping individual differences in the aspect of number sense as the ability to pinpoint the position of a number on the number line in fourth grade. The evaluation of the parameters of all theoretical models showed that during primary school the second analyzed aspect of number sense - the non-symbolic magnitude estimation ability is not related to the causal relationship with the success in learning mathematics.

\section{Discussions}

In the current article, we focused on the relationship between number sense and success in learning mathematics in a two-year longitudinal study.

According to the results of the correlation analysis, by fourth grade of primary school, the relationship between the success in learning mathematics and number sense as the non-symbolic magnitude estimation ability is weaker than in third grade. A similar trend was detected for nonverbal intelligence. These results are consistent with the studies of Russian schoolchildren reporting a gradual reduction of the role of multiple cognitive characteristics contributing to academic success in school years, from the speed of information processing to nonverbal intelligence [6]. In particular, it was shown that number sense as the non-symbolic magnitude estimation ability and the annual grades in mathematics are closely related in primary school.

On the contrary, number sense measured as the ability to accurately determine the position of a number on the number line is more related to the success in learning mathematics in fourth grade than in third grade. In other words, the longer children study in primary school, the less their success in learning mathematics depends on the non-symbolic magnitude estimation ability and, to a greater extent, on the number sense aspect associated with symbolically expressed quantities. At the same time, the interpretation of the obtained results is also valid in the opposite direction: in fourth grade, in comparison to third grade, the level of development of the number sense aspect associated with non-symbolically expressed quantities to a lesser extent depends on the success in learning mathematics, and to a 
greater extent on the number sense aspect related to operating with numerical material. Such multidirectional tendencies in the interrelationship of success in mathematics with the perceptions of numbers in nonsymbolic and symbolic systems are related to the specifics of the learning process in primary school, in particular with the content of the school mathematics course in Russia [13].

In the cross-lagged modeling, we compared four competing models of the relationship of the two aspects of number sense and success in learning mathematics. According to the analysis, the reciprocal model best described the empirical data suggesting that there are cross-lagged associations between number sense and success in learning mathematics in primary school even after controlling for nonverbal intelligence. The estimation of the cross-lagged associations within the model showed that the only significant association was the one between success in learning mathematics in third grade and number sense measured as the ability to determine the position of a number on the line in fourth grade. In other words, the accuracy of determining the position of a number on a number line depends on the success of mastering the concept of number and arithmetic operations in primary school: the higher were students' marks in third grade, the better they performed in fourth grade on the number sense tasks on symbolic magnitude estimation. On the contrary, for the number sense aspect associated with the non-symbolic magnitude estimation ability by the end of primary school, the success in learning mathematics measured at an earlier age was statistically insignificant.

These differences for the two indicators of number sense indicated a significant effect of training on the accuracy of determining the position of a number on the number line even in the analysis of modified models, which is consistent with previous studies [17]. According to research, number sense is a multidimensional construct in which low- and high-level characteristics can be distinguished [4]. As a rule, lowlevel characteristics are associated with the ability to estimate and operate with non-symbolically expressed quantities and are cosidered as evolutionarily preserved traits, and high-level characteristics are related to the symbolic representation of objects which is developed in the course of education. The lack of clear boundaries between number sense, especially associated with symbolic magnitude estimation, and mathematical skills leads to the question of what determines success in the Number Line task: the level of mastering the educational program in mathematics or number sense.

However, as described above, non-symbolic and symbolic magnitude estimation is based on the same principles, which makes it possible to analyze both test tasks as diagnostic for number sense but they should be considered as more (in the case of the Number Line) or less related to arithmetic skills [4]. From this point of view, the results obtained in this paper on the role of the success in learning mathematics in third grade in the formation of individual differences in number sense measured as the ability to determine the position of a number on the line look logical: the better the school curriculum is mastered in third grade, including knowledge of the numbers up to 1000 , the more precise is the performance on the Number Line task. An additional argument is the tendency of the relationship between academic success and the accuracy of determining the position of a number on the line to strengthen by the end of the primary school, as revealed in the correlation analysis. Thus, as arithmetic knowledge and skills develop, children begin to use more accurate linear representations rather than logarithmic ones [3].

We found no cross-lagged associations between the number sense aspect related to non-symbolic magnitude estimation and the success in learning mathematics. This result is consistent with previous evidence on the lack of prognostic associations between number sense and mathematical success, e.g. at primary school age (see [5]).

In the course of the analysis, differences were also revealed in the dynamics of the two aspects of number sense at primary school: the number sense aspect associated with non-symbolic magnitude estimation was the most stable in time. This result is consistent with the available data on the differences in the temporal stability of the two analyzed aspects of number sense $[13,20]$. The observed differences in dynamics can also determine the specific relationship between the various indicators of number sense and the success in learning mathematics.

It should be emphasized that according to the results of this study, none of the number sense aspects measured in third grade significantly contributed to the variation of success in learning mathematics by the end of primary school, which is different from the results obtained on other samples. Thus, in most longitudinal studies, the indicators of number sense associated with the estimation of quantities in symbolic and non-symbolic systems are considered as pre-school predictors of mathematical success at school [7, 16].

The discrepancies in the results might be related to the specifics of the Russian education system, in particular, to the evaluation of the success in learning the school disciplines. As a rule, in the Russian education system, teacher's assessment is the only criterion for assessing the academic success of junior schoolchildren and is based on a subjective vision of the material learned by a child based on a set of tests and individual answers in class throughout the year. In studies involving schoolchildren, for example, from the United States, Great Britain and Western Europe, academic success is also assessed by a teacher but based on the final standardized test assignments, which include not only educational material, but also tasks that require non-standard solutions that are not explained during the school year and the ability to apply the acquired knowledge in real life $[5,8]$. For example, in the study on a Russian sample of senior students, only moderate correlations were found (from 0.41 to $0.56, p<0.01$ ) between three different indicators of academic success in mathematics in ninth grade -- teacher assessment, the state exam and a computerized test [6]. Such discrepancies in the indicators of academic success 
might be the reason of changes in the relationship between number sense and the success in learning mathematics observed in the results of previous studies.

At the same time, the obtained data on the causeeffect relationship of number sense and the success in learning mathematics in primary school may reflect the zonal nature of the interaction of psychological and nonpsychological factors, i.e. a variety of cognitive characteristics and indicators of academic success in this study [23]. Such relationship can only be characteristic of the initial stage of schooling when the foundations of mathematical knowledge essential for operating with symbolically expressed quantities are acquired. In a number of studies, it is reported that number sense measured at primary school age is a predictor of later mathematical success measured at the middle or high school age $[8,24]$. In this paper, both number sense and the success in learning mathematics were measured in primary school. Perhaps other results would be obtained if number sense measured in primary school was analysed as an earlier predictor of later academic success. However, the verification of this hypothesis lied outside the scope of this paper and will be the subject of a further longitudinal study.

Thus, in this paper it was shown that the learning process can influence the formation of cause-effect relationships between indicators of cognitive functioning and academic success in mathematics. In the context of the relationship between cognitive development and learning, a learning effect was found for the number sense aspect measured as the ability to accurately indicate the position of a number on the line. For the number sense aspect related to non-symbolic magnitude estimation, the success in learning mathematics measured a year earlier did not play an important role. The obtained results highlight the importance of studying the influence of national educational systems on individual psychological development and necessitate a cross-cultural longitudinal analysis of the interaction of cognitive functioning and learning throughout the school age.

This study was supported by the grant from the Russian Science Foundation [grant RSF №17-78-30028].

\section{References}

1. T.N. Tikhomirova, Procedia - Social and Behavioral Sciences, 237, 1260 (2017)

2. M. Rodic, X. Zhou, T. Tikhomirova, W. Wei, S. Malykh, V. Ismatulina, E. Sabirova, Y. Davidova, M. Tosto, J-P. Lemelin, Y. Kovas, Developmental Science, 18, 165 (2015)
3. D.C. Geary, Developmental psychology, 47, 1539 (2011)

4. S. Dehaene, The Number Sense: How the mind creates mathematics (Oxford University Press USA, Oxford, 2011)

5. T.N. Tikhomirova, S.B. Malykh, M.G. Tosto, Y.V. Kovas, Psychological journal, 35, 41 (2014)

6. T.N. Tikhomirova, S.B. Malykh, Cognitive bases of individual differences in academic achievement (Nestor-History, Moscow, 2017)

7. A. Desoete, A. Ceulemans, F. de Weerdt, S. Pieters, Brit. J. Educ. Psychol, 82, 64 (2012)

8. J. Halberda, M.M. Mazzocco, L. Feigenson, Nature, 455, 665 (2008)

9. M. Inglis, S. Batchelor, C. Gilmore, Psychon. Bull. Rev., 18, 1222 (2011)

10. S.B. Malykh, I.A. Lysenkova, I.M. Zakharov, T.N. Tikhomirova, The European Proceedings of Social \& Behavioural Sciences, 33, 235 (2017)

11. F. Soltész, D. Scőz, L. Scőz, Behav. Brain Funct., 6, 1 (2010)

12. S.B. Malykh, T.N. Tikhomirova, C. Zhou, W. Wei, M. Rodic, E.B. Misozhnikova, Y.A. Davydova, Y. Kovas, Voprosy psihologii, 5, 133 (2012)

13. J.E. Opfer, R.S. Siegler, Cogn. Psychol., 55, 169 (2007)

14. T.N. Tikhomirova, E.B. Misozhnikova, Y.V. Kuzmina, S.B. Malykh, SHS Web of Conferences, 10, 02007 (2017)

15. T.D. Little, K.J. Preacher, J.P. Selig, N.A. Card, International Journal of Behavioral Development, 31, 357 (2007)

16. M.M. Mazzocco, L. Feigenson, J. Halberda, PLoS ONE, 6, 1 (2011)

17. R.S. Siegler, J.L. Booth, Child dev., 75, 428 (2004)

18. R.S. Siegler, G.B. Ramani, J. Educ. Psychol., 101, 545 (2009)

19. R.S. Siegler, Y. Mu, Psychological Science, 19, 759 (2008)

20. J. Halberda, R. Ly, J. Wilmer, D. Naiman, L. Germine, Proc. Natl. Acad. Sci., 109, 11116, (2012)

21. M.G. Tosto, T. Tikhomirova, E. Galajinsky, K. Akimova, Y. Kovas, Procedia - Social and Behavioral Sciences, 86, 423 (2013)

22. J. Raven, Cognitive Psychology, 41, 1 (2000)

23. T.N. Tikhomirova, A.D. Modyaev, N.M. Leonova, S.B. Malykh, Psychological journal, 36, 43 (2015)

24. J.L. Booth, R.S. Siegler, Child dev., 79, 1016 (2008) 